\title{
Diversity of Emergency Codes in Hospitals
}

\author{
Nilsa Padilla-Elías ${ }^{1}$, Marisol Peña-Orellana ${ }^{1,3}$, Ralph Rivera-Gutiérrez ${ }^{1,3}$, Juan A. Gónzalez-Sánchez ${ }^{2}$, \\ Heriberto A. Marín Centeno ${ }^{1,3}$, Héctor Alonso-Serra ${ }^{1}$, Liza Millán-Pérez ${ }^{1}$, Patricia Monserrrate-Vázquez ${ }^{1}$ \\ ${ }^{1}$ (Center for Public Health Preparedness) Graduate School of Public Health, Medical Sciences Campus, University of Puerto Rico, \\ San Juan, Puerto Rico; ${ }^{2}$ (School of Medicine) Department of Emergency Medicine, Medical Sciences Campus, University of Puerto \\ Rico, San Juan, Puerto Rico; ${ }^{3}$ Department of Health Services Administration, Graduate School of Public Health, Medical Sciences \\ Campus, University of Puerto Rico, San Juan, Puerto Rico. \\ Email: nilsa.padilla@upr.edu
}

Received September $13^{\text {th }}, 2013$; revised October $14^{\text {th }}, 2013$; accepted November $2^{\text {nd }}, 2013$

Copyright (C) 2013 Nilsa Padilla-Elías et al. This is an open access article distributed under the Creative Commons Attribution License, which permits unrestricted use, distribution, and reproduction in any medium, provided the original work is properly cited.

\begin{abstract}
Background: Hospitals must be prepared to deal efficiently and effectively with different emergencies. To accomplish this, several countries have standardized their hospital emergency codes to improve their response capability. This is particularly important in Puerto Rico given that many health professionals, particularly physicians and nurses, provide services in more than one hospital. This study examined the emergency codes and alerts utilized in Puerto Rican hospitals. Objective: To assess hospitals' level of emergency preparedness and response capability related to the variability of emergency codes and alerts utilized to respond to a situation in Puerto Rico. Method: A survey was conducted to characterize hospital emergency department level of preparedness and response to a mass fatality incident. A total of 39 out of a sample of 44 hospitals participated in the study. Semi-structured questionnaires were administered by the research team to members of each hospital's administrative staff to explore the following: general hospital characteristics, emergency plans, emergency department capacity, collaborative agreements, personnel training, emergency communications, laboratory facilities, treatment protocols, security, epidemiologic surveillance, equipment and infrastructure. Results: Some hospitals in Puerto Rico use color coded emergency alerts, while others use key words or codes. Single color emergency codes can have different meanings in different hospitals. Conclusions: The findings clearly show that there is a lack of uniformity and clarity in the emergency codes utilized by hospitals in Puerto Rico. Single color codes have diverse meanings in different hospitals. This could adversely affect hospitals' efficient and effective emergency response.
\end{abstract}

Keywords: Communications; Hospitals Health; Health Communications; Emergency Preparedness; Preparedness; Emergency Codes

\section{Introduction}

An emergency is defined as any incident, caused by humans or a natural event, that requires an effective, responsive action to protect life or property [1]. Therefore, the response to an emergency must be quickly, coordinated and well-planned [2]. Initial efforts for the standardization of emergency codes in hospitals started with an incident where three persons were killed in southern California in a shooting at a medical center after the wrong code was called [3]. This particular incident led the Hospital Association of Southern California to develop a comprehensive campaign to achieve standardizetion of hospital emergency codes [1].

Assuring emergency preparedness and response re- quires a systematic and structured methodology that enables an objective assessment [4]. As part of this effort, a number of jurisdictions have moved towards standardization of hospital emergency codes. The need for code uniformity is underscored due to the mobility of the health care workforce. Staff who are reassigned to a new medical facility, or who must practice in more than one facility, need to be immediately familiarized with a code identifying the nature of a given crisis and their expected response [5].

The purpose of the study was to assess hospitals' level of emergency preparedness and response capability related to the variability of emergency codes and alerts utilized to respond to a situation of emergency in hospitals in Puerto Rico. 


\section{Methods}

A survey was conducted to characterize hospital emergency department level of preparedness and response to a mass fatality incident. A total of 39 out of a sample of 44 hospitals participated in the study. Semi-structured questionnaires were administered by trained research assistants to hospital directors to explore the following: general hospital characteristics, emergency plans, emergency department capacity, collaborative agreements, personnel training, emergency communications, laboratory facilities, treatment protocols, security, epidemiologic surveillance, equipment and infrastructure. The selection of the hospital emergency departments that participated in the study was conducted by the Puerto Rico Department of Health (PRDOH) Office of Public Health Preparedness and Response (OPHPR). The OPHPR provided the research team with a list of 44 healthcare facilities grouped into six (6) coalitions: North, South, East, West, Metro A and Metro B. The list consisted of forty-one (41) hospitals and three (3) community health centers, which included a Diagnostic and Treatment Center, a primary health center and a family health center. All these healthcare facilities were located in 23 municipalities throughout Puerto Rico. Figure 1 shows the location of the participating emergency departments. At the end of the study, 39 hospital facilities agreed to participate in the study, yielding a response rate of $88.6 \%$.

To gather the data, seven (7) electronic instruments were constructed considering ten (10) dimensions identi- fied through the literature. These dimensions were: 1 ) general characteristics of the hospital; 2) emergency plans; 3) collaborative agreements between agencies; 4) infrastructure and equipment; 5) epidemiologic surveillance; 6) protocols for medical treatment; 7) laboratory; 8) training among personnel; 9) communications during an emergency; and 10) hospital physical security. Confidentiality and voluntary participation issues were discussed with all subjects and all study activities were reviewed and approved by the Human Subjects Institutional Review Board. IRB: RCM IRB, protocol \#A66640211, January 31, 2011.

\section{Results}

Results from this study showed that some hospitals use color coded emergency alerts, while others use key words or codes. Moreover, a single color emergency code can have diverse meanings in different hospital installations. Among the key colors that showed the highest diversity were code blue with seven different meanings, code yellow and code white with six different meanings, and code green with five. The findings of the study showed the following color codes as the most used: red for fire with $79.5 \%(\mathrm{n}=31)$; gray for safety/security situation with $74.4 \%(n=29)$; and, green for cardio-respiratory arrest with $71.8 \%(n=28)$. Figure 2 shows the different emergency codes used in hospitals in Puerto Rico based on the response of 39 healthcare facilities.

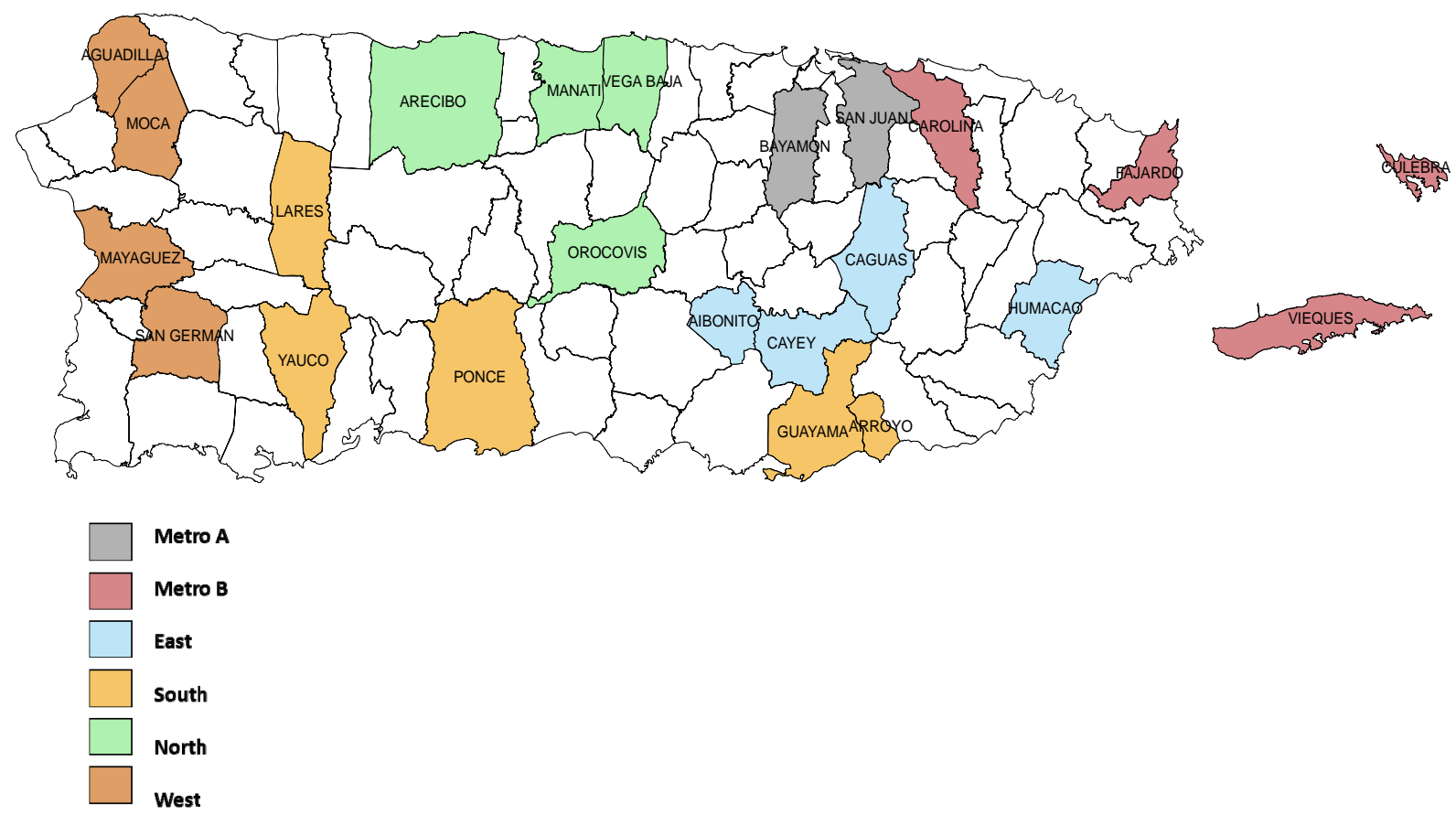

Source: Office of Public Health Preparedness and Response, Puerto Rico Department of Health

Figure 1. Location of Participating Emergency Departments. 
Figure 2. Diversity in Puerto Rico hospital's use of emergency codes $\mathbf{N}=39$.

\begin{tabular}{|c|c|c|c|}
\hline Emergency Code & Meaning of the code & $\mathbf{n}$ & $\%$ \\
\hline \multirow{5}{*}{ Green } & Cardio-respiratory arrest & 28 & 71.8 \\
\hline & Death or health hazard & 1 & 2.6 \\
\hline & Agitated patient & 1 & 2.6 \\
\hline & Internal disaster & 1 & 2.6 \\
\hline & Rapid Response Team & 1 & 2.6 \\
\hline \multirow{3}{*}{ Black } & Assisting patient with primary need & 8 & 20.5 \\
\hline & Bomb (explosives or threat) & 1 & 2.6 \\
\hline & Dead or dying patient & 1 & 2.6 \\
\hline \multirow{2}{*}{ Orange } & Rapid Response Team & 8 & 20.5 \\
\hline & Critically ill patient & 2 & 5.1 \\
\hline Brown & Evacuation & 10 & 25.6 \\
\hline \multirow{7}{*}{ Blue } & External disaster & 11 & 28.2 \\
\hline & External/Internal disaster & 5 & 12.8 \\
\hline & Cardio-respiratory arrest & 5 & 12.8 \\
\hline & Disaster & 4 & 10.3 \\
\hline & Flood & 2 & 5.1 \\
\hline & Earthquake & 2 & 5.1 \\
\hline & Internal disaster & 1 & 2.6 \\
\hline \multirow{3}{*}{ Red } & Fire & 31 & 79.5 \\
\hline & Arrival of first victim & 1 & 2.6 \\
\hline & Major emergency & 1 & 2.6 \\
\hline \multirow{6}{*}{ White } & Flood & 9 & 23.1 \\
\hline & End of the emergency & 2 & 5.1 \\
\hline & Kidnapping/robbery/theft of child/infant or child lost & 1 & 2.6 \\
\hline & Puncture with syringe & 1 & 2.6 \\
\hline & Emergency with psychiatricpatient & 1 & 2.6 \\
\hline & Bomb (explosives or threat) & 1 & 2.6 \\
\hline \multirow{6}{*}{ Yellow } & Spill of hazardous material/substance & 14 & 35.9 \\
\hline & Safety/security situation & 2 & 5.1 \\
\hline & Kidnapping/robbery/theft of child/infant or child lost & 2 & 5.1 \\
\hline & External disaster & 2 & 5.1 \\
\hline & Emergency with pediatric patient & 1 & 2.6 \\
\hline & Internal disaster & 1 & 2.6 \\
\hline \multirow{3}{*}{ Pink } & Kidnapping/robbery/theft of child/infant or child lost & 9 & 23.1 \\
\hline & Fire & 1 & 2.6 \\
\hline & Emergency with pediatric patient & 1 & 2.6 \\
\hline \multirow{4}{*}{ Violet } & Network shut down & 1 & 2.6 \\
\hline & Water & 1 & 2.6 \\
\hline & Hospital at maximum capacity & 1 & 2.6 \\
\hline & Kidnapping & 1 & 2.6 \\
\hline \multirow{4}{*}{ Grey } & Safety/security situation & 29 & 74.4 \\
\hline & External disaster & 2 & 5.1 \\
\hline & Guns or fire arms & 1 & 2.6 \\
\hline & Escaped Alzheimer patient & 1 & 2.6 \\
\hline
\end{tabular}




\begin{tabular}{cccc} 
Continued & & & \\
\hline Emergency Codes & Meaning of the code & n & \% \\
\hline 1014 & Suspicious person & 1 & 2.6 \\
Alpha & Kidnapping/robbery/theft of child/infant or child lost & 1 & 2.6 \\
Amber & Kidnapping/robbery/theft of child/infant or child lost & 11 & 28.2 \\
Coca Cola & Real patient during drill & 1 & 2.6 \\
D1 & External disaster & 1 & 2.6 \\
Delta & External disaster & 1 & 2.6 \\
Delta & Internal disaster & 1 & 2.6 \\
H2O & Flood & 20 & 51.3 \\
H2O & Spilled liquids & 1 & 2.6 \\
Don't know & Don’t know the emergency codes & 3 & 7.7 \\
"Rápida" & Rapid Response Team & 1 & 2.6 \\
S & Attempted kidnapping & 2 & 5.1 \\
TD & Evacuation & 1 & 2.6 \\
\hline
\end{tabular}

\section{Discussion}

The findings clearly show that there is a lack of uniformity and clarity in the emergency codes and alerts utilized in hospitals in Puerto Rico. A single code could have diverse meanings in different hospitals. This could adversely affect an efficient and effective emergency mobilization of patients, visitors and hospital personnel during an emergency. The lack of standardization increases the potential for confusion or misinformation during critical times [1]. In Puerto Rico, on August 10, 2011 Law 1702011, which allows the Department of Health to implement the standardization of protocol codes for emergency care facilities in the private and public health sector was approved [6]. This law represents an additional preparedness effort; however, it is not completely implemented yet.

An emergency can happen at any time. Clear communication is a key element to ensure a quick response to protect patients, visitors and staff. The fact is that code alert and standardization among all hospitals may not be immediate and there will need to be a planned transition to the recommended code set. Several implications of the diversity or inconsistent codes for the differences in terminology have to be considered for planning, communication, and operations during an actual event [7]. According to the Hospital Incident Command System guidance, it is important to point out the need to use clear language in case of a disaster event, especially when dealing with external resources [8]. The goal is for hospitals to phase in the implementation of the recommended codes so that the required materials and training can be developed and offered at a time best suited for hospital personnel. Clearly, considerable training and financial resources will be required for this transition to be conducted efficiently and effectively [9].

\section{Acknowledgements}

Data for this study came from the Assessment of the
Emergency and Disaster Preparedness and Response Capabilities of Hospital Emergency Departments in Puerto Rico, conducted during 2011. Funding source by ASPRUSDHHS, administered by the Puerto Rico Department of Health, Office of Public Health Preparedness and Response, under contract 2010-DS0609. We acknowledge the support we received from the hospitals that participated in the study and the Hospital Association of Puerto Rico for their willingness to take into account data derived from our studies in formulating health policy. We would also like to thank Wined Ramírez López, for helping in the edition of this manuscript and Julieanne Miranda Bermúdez, for the preparation of the map.

\section{REFERENCES}

[1] California Hospital Association, "Hospital Emergency Code Standardization Survey,” 2011.

[2] A. Truesdell, "Meeting Hospital Needs for Standardized Emergency Codes-The HASC Response," Journal of Health Care Prot Manage, Vol. 21, No. 1, 2005, pp. 7789.

[3] Hospital Association of Southern California, "Healthcare Emergency Codes: A Guide for Code Standardization,” 2009.

[4] B. Adini, A. Goldberg, D. Laor, R. Cohen, R. Zadok and Y. Bar-Dayan, "Assessing Levels of Hospital Emergency Preparedness,” Prehospital and Disaster Medicine, Vol. 21, No. 6, 2006, p. 7.

[5] Ministry of Health Services Policy Communiqué, "Standardized Hospital Colour Codes,” 2011.

[6] Commonwealth of Puerto Rico, "Act 170 to Establish the Universal Protocol Emergency Codes for in Public and Private Health Facilities,” 2011.

[7] M. McMahon, "The Many Codes for a Disaster: A Plea for Standardization,” Disaster Management \& Response, Vol. 5, No. 1, 2007, pp. 1-2. http://dx.doi.org/10.1016/j.dmr.2006.12.001 
[8] California Emergency Medical Services Authority, "The Hospital Incident Command System (HICS) Guidebook,” 2006. www.emsa.ca.gov/HICS.
[9] WHA Medical and Professional Affairs Council, "Wisconsin Hospital Standardized Alert Code Recommendations,” 2011. 